\title{
Capturing User Intent for Information Retrieval
}

\author{
Hien Nguyen \\ Computer Science and Engineering Department \\ University of Connecticut \\ 371 Fairfield Rd., Unit 2155 , Storrs, CT 06269-1155 \\ hien@cse.uconn.edu
}

\begin{abstract}
We study the problem of employing a cognitive user model for information retrieval in which knowledge about a user is captured and used for improving retrieval performance and user satisfaction. In this proposed research, we improve retrieval performance and user satisfaction for information retrieval by building a user model to capture user intent dynamically through analyzing behavioral information from retrieved relevant documents, and by combining captured user intent with the elements of an information retrieval system. We use decision theoretic principles and bayesian networks for building this model. The novelties of our approach lie with the fine-grained representation of the model, the ability to learn user knowledge incrementally and dynamically, the integration of user intent and system elements for improving retrieval performance and the unified evaluation framework to assess the accuracy of user intent captured and effectiveness of our model.
\end{abstract}

\section{Problem Statement}

We study the problem of employing a cognitive user model for information retrieval in which knowledge about a user is captured and used for improving retrieval performance and user satisfaction. The problem of employing a user model for information retrieval has been investigated since the late 80s (Brajnik, Guida, \& Tasso 1987) to address the difficulty of traditional information retrieval which is to satisfy a user's information needs by retrieving information with good quality quickly. It has many applications from information filtering, and text recommendation systems (e.g (Balabanovic 1998; Billsus \& Pazzani 2000)). This problem is wellknown for the challenges it poses to Information Retrieval (IR), and User Modeling (UM) communities. We have identified four primary challenges of this problem which are (i) non-observability of user knowledge (ii) uncertainty in modeling user interactions (ii) vagueness of a user's information needs (iv) and dynamics of user knowledge which changes over time as a result of new information, and temporal factors. These challenges come from the main problem of modeling a user in information seeking. Unfortunately, traditional IR does not offer a way to overcome these challenges

Copyright (C) 2004, American Association for Artificial Intelligence (www.aaai.org). All rights reserved. because its framework supported very little users' involvement (Saracevic 1996). The thesis of this proposed research is to improve retrieval performance and user satisfaction for $I R$ by building a user model to capture user intent dynamically through analyzing behavioral information of retrieved relevant documents, and combine it with the elements of an IR system. Our methodologies for this research are (i) to combine system-centered approaches and user-centered approaches by taking advantage of well-established evaluation frameworks in IR, and using the strength of knowledge representation techniques in artificial intelligence (AI) to build this model. As pointed out in (Saracevic, Spink, \& Wu 1997), so far there has been a little crossover between IR and AI communities with regards to building user models for IR; and (ii) to create an unified evaluation framework which combines IR procedures with human computer interaction (HCI) usability testing to evaluate this model. We use decision theoretic (DT) principles and Bayesian networks (BNs) for building this model because the process of elicitation of user's preferences has long been studied in decision theory (Keeney \& Raiffa 1976). BNs (Pearl 1988) offer a mathematically sound and intuitive representation to capture uncertainty which is a key factor in modeling a user. We seek to address the following questions:

- What factors of user intent should be captured? How can we capture, represent and use them for improving retrieval performance and user satisfaction?

- How can we combine user intent with elements of an IR system to maximize retrieval performance and user satisfaction?

- How do we evaluate the accuracy, and the effectiveness of our user model?

\section{Our approach}

\section{User model architecture}

We hypothesize that by decomposing user intent into 3 components: interests, preferences and context, our user model will help an IR system better adapt to the user than a model containing each factor alone. We refer to our user model as the IPC user model (Santos, Nguyen, \& Brown 2001; Santos et al. 2003a; 2003b). In our model, we capture, represent and use the information on what a user is currently interested in (the Interests); how a query needs to be 
constructed (the Preferences) and why the user dwells on a search topic (the Context) to modify a user's queries proactively. We capture the Context, the Interests, and the Preferences aspects of a user's intent with a context network $(C)$, an interest set $(I)$, and a preference network $(P)$. A context network $(\mathrm{C})$ is a directed acyclic graph (DAG) that contains concept nodes and relation nodes. We construct $C$ dynamically by finding the intersections of all retrieved relevant documents. Each element of I consists of an interest concept and interest level. We use a spreading activation algorithm on $C$ to reason about a new $I$. We use a Bayesian network to represent $P$ because of its expressiveness, and power of modeling uncertainty. $P$ is used to represent a user's preferences over a class of tools used to modify a query. $P$ is updated when a user gives feedback based on the frequency of tools used in the past. An original query graph is modified by adding relation and concept nodes from $C$ given current $I$ and tools inferred by $P$. In summary, $C$ contains the knowledge used for modifying a user's query while $I$ and $P$ contain information to determine which knowledge should be included. In other words, we disambiguate a user's information needs by using information from $I, P, C$.

\section{Evaluation}

The main questions needed to be answered in this evaluation are: (1) How accurate is the user intent that has been captured? (2) How competitive is the improvement obtained by using our user model compared with the improvement obtained by existing approaches? and (3) How effective is our user model is in terms of improving user satisfaction? In our initial study (Santos et al. 2003b), we evaluate the effects of our user model with regards to retrieval performance using a medical database with 521 records, over a set of 35 queries. This evaluation showed that precision and recall of the system with a user model are at least as good as without a user model. In some cases, it is actually better. In the pilot usability testing (Santos et al. 2003b), however, we were unable to find a significant effect of the user model on user or task efficiency. The main reasons are (i) small database and (ii) opaqueness of the system's behaviors.

\section{Future work}

Our user model helps the target IR system adapt to the user's interests, preferences and context. Some difficulties may arise such as: degrading in retrieval performance due to a user's poor domain knowledge and difficulty in retrieving new information which contradicts with a user's interests. Fortunately, the target IR system consists of some elements containing built-in domain and system knowledge (e.g indexing scheme). Therefore, we propose to combine user intent and the elements of an IR system in a decision theoretic framework for improving retrieval performance and user satisfaction. The raw architecture of the proposed model takes user intent (IPC model), elements of an IR system, and the goal representing a user's purpose of using the IR system (e.g recall-driven or precision-driven) as inputs and formulates a decision tree which can be used to predict what is the best move for the user. The challenges for this task include (i) which attributes and values should be included and (ii) how to determine utility functions to maximize the effectiveness of our user model. We compare the effectiveness of our user model approach against Ide dec-hi with term frequency inverted document frequency using popular collections in IR such as CRANFIELD, NPL, MEDLINE, CACM, and TREC disks. The challenge here is to maintain the consistency, scalability and robustness of our approach for both small and large collections. Lastly is the extension of our usability testing which uses the results from the pilot study in (Santos et al. 2003b). The novelty of our future work is the combination of system-centered and user-centered approaches for representation and evaluation of our user model.

Acknowledgement This work was supported in part by the Advanced Research and Development Activity (ARDA)-U.S. Government. Any opinions, findings and conclusions or recommendations expressed in this material are those of the authors and do not necessarily reflect the views of the U. S. Government

\section{References}

Balabanovic, M. 1998. Exploring versus exploiting when learning user models for text recommendation. User Modeling and User-Adapted Interaction 8:71-102.

Billsus, D., and Pazzani, M. J. 2000. User modeling for adaptive news access. Journal of User Modeling and UserAdapted Interaction 10:147-180.

Brajnik, G.; Guida, G.; and Tasso, C. 1987. User modeling in intelligent information retrieval. Information Processing and Management 23(4):305-320.

Keeney, R. L., and Raiffa, H. 1976. Decision with Multiple Objectives: Preferences and Value Tradeoffs. John Wiley and Sons.

Pearl, J. 1988. Probabilistic Reasoning in Intelligent Systems: Networks of Plausible Inference. Morgan Kaufmann, San Mateo, CA.

Santos, E.; Nguyen, H.; Zhao, Q.; and Hua, W. 2003a. User modelling for intent prediction in information analysis. In Proceedings of the HFES-03, 1034-1038.

Santos, E.; Nguyen, H.; Zhao, Q.; and Pukinskis, E. 2003b. Empirical evaluation of adaptive user modeling in a medical information retrieval application. In Proceedings of the ninth User Modeling Conference, 292-296.

Santos, E.; Nguyen, H.; and Brown, S. M. 2001. Kavanah: An active user interface information retrieval application. In Proceedings of 2nd Asia-Pacific Conference on Intelligent Agent Technology, 412-423.

Saracevic, T.; Spink, A.; and Wu, M. 1997. Users and intermediaries in information retrieval: What are they talking about? In Proceedings of the 6th International Conference in User Modeling, 43-54. Springer-Verlag Inc.

Saracevic, T. 1996. Relevance reconsidered. In Proceedings of the Second International Conference on Conceptions of Library and Information Science: Integration in Perspective. Copenhagen: The Royal School of Librarianship, 201-218. 